\title{
Behavior of Magnetic Moment in a New CoMnTiSn(Sb) Quaternary Heusler Alloy
}

\author{
*Osafile, O.E. \& Azi, J.O. \\ Department of Physics \\ Federal University of Petroleum Resources \\ PMB 1221, Effurun, Nigeria \\ E-mail:osafile.omosede@fupre.edu.ng
}

\begin{abstract}
The quaternary Heusler alloys is a relatively new equal atomic weight material under research for gainful applications in exotic devices from spintronics to energy conversion efficiency. By selecting $3 d$ transition metals with the valency carefully considered, we present results from a first principles calculation using the generalized gradient approximation (GGA) via density functional theory (DFT) as implemented in the quantum ESPRESSO code for CoMnTiSn(Sb) quaternary alloys. Upon structural optimization, both alloys crystallize in the type-Y1 state. Both alloys display a unique behavior variant from the expected SlaterPauling rule of $\left(M_{t}=Z_{t}-24\right)$ applicable to quaternary alloys. Hence, CoMnTiSn behaves as a ferromagnetic spin gapless semiconductor whereas CoMnTiSb behaves as a non-magnetic semiconductor with a band gap of $0.40 \mathrm{eV}$. The elastic properties describe them as hard but ductile materials with CoMnTiSn having a higher ductility (B/G ratio) of 2.43. The anisotropic test also records a $65 \%$ rate resistance to deformation in CoMnTiSn. We believe that these materials have applications in semiconductor spintronics devices with slight tuning.
\end{abstract}

Keywords: Density functional theory, quaternary Heusler alloy, elastic constants.

Aims Research Journal Reference Format:

Osafile, O.E. \& Azi, J.O. (2018): Behavior of Magnetic Moment in a New CoMnTiSn(Sb) Quaternary Heusler Alloy.

Advances in Multidisciplinary Research Journal. Vol. 2. No. 4, Pp 89-99

\section{INTRODUCTION}

Heusler alloys have become ubiquitous in the field of condensed matter physics, especially in materials, and find application in spin electronics, energy conversion efficiency and many other fields. For some time, many materials such as GaAs [1-3] seemed to have been adequate, however, continuous research revealed the need for materials with tunable (structural, magnetic and electronic) properties. In this respect, the various variants of the Heusler alloys have proven to be quite invaluable and progressive in terms of promising results obtained via theoretical (first principle calculations using density functional theory) and experimental research. Ever since the half-metallic ferromagnet (HMF) was first studied by Groot et al [4], much attention has been paid to the Heusler family because among other half metallic materials such as magnetic oxides [5] and dilute magnetic III-V semiconductors, they have a higher Curie temperature [6] and hence, better efficiency.

Many elemental, structural, electronic and transport properties contribute significantly to the usefulness of the vast Heusler family in its various areas of application, especially in spintronics. 
A family of the Heusler alloy of recent interest is the quaternary Heusler alloy with the $1: 1: 1: 1$ stoichiometry. The quaternary Heusler alloy has a stoichiometric composition of XX'YZ of space group $F \overline{43} m$ (X, $X^{\prime}$, and $Y$ are transition metals, whereas $Z$ is a main-group element). Typically, the valence of $X$ is higher than the valence of $X$, and the valence of the $Y$ element is lower than the valence of both $X$ and $X^{\prime}$ and such compounds crystallize in the $Y$ type (prototype LiMgPdSn) [7,8] structure. This variant was theoretically predicted by Wang in 2008 [9]. The usual method for conceiving this variant has been to combine two ternary alloys. Wang predicted that they have a half-metallic nature with a high Curie temperature [10].

The work of Ouardi et al. [11] revealed what is probably a new variant of half-metallic ferromagnetic (HMF) Heusler materials termed spin gapless semiconductors (SGS). They proposed that these emerging materials are vital in semiconductor spintronic devices. These materials possess a zero band gap for one spin channel and a band gap in the other channel. They proposed the possibility of SGS serving as a bridge between HMF and semiconductors. Some of their desirable properties include spin polarized currents resulting from holes and electrons, switch-ability between $n$ and $p$ type spin polarized carriers in an electric field, and the requirement of almost no energy to excite electrons from the valence band to the conduction band. Wang [9] theoretically predicted their existence and ever since, they have received intense research interest. Due to their unique band structure, SGS materials are considered potentially better substitute candidates for diluted magnetic semiconductors (DMS). Many Heusler alloys are found to possess the SGS band structure as revealed by ab -initio calculations [12, 13]. Furthermore, it has been experimentally confirmed in a few alloys such as $\mathrm{Mn}_{2} \mathrm{CoAl}$ [14], CoFeMnSi [15] and CoFeCrGa [15]. Very recently, thin films of $\mathrm{Mn}_{2} \mathrm{CoAl}$ were synthesized to determine its applicability in the devices [16-18].

In addition, some other quaternary Heusler alloys, CoFeMnX (X = Al, Si, Ga, Ge) [17, 19-21] have recently been synthesized successfully. Ab -initio calculations and experimental observations indicate that there is a great possibility of achieving half-metallicity in these alloys. Klaer et al. [22] predicted halfmetallic behavior in the case of CFMG. These classes of alloys, require almost negligible energy to excite electrons from the valence band to the conduction band. This is due to their unique near zero band gap and total spin polarization, which enables them to switch between $n$ and $p$ type carriers by the application of an electric field. These materials have important applications in spintronics and thermoelectrics.

Research is ongoing in the field of quaternary alloy. Hence, in this work, we pay attention to novel materials CoMnTiX $(X=S b, S n)$. Co based alloys have a high value of spin polarization at low temperatures [23, 24]. Our report is on the structural, magnetic, mechanical, and electronic properties of both alloys. The work is motivated by theoretical and experimental results for quaternary alloys such as CoMnTiAl, FeMnTiAl, NiFeMnGa, NiCoMnGa and CoFeMnZ (Z = Al, Si, Ga, Ge) [10, 25, 26]. We found CoMnTiSb to be a non-magnetic semiconductor and CoMnTiSn to be nearly a p-type spin gapless semiconductor, which promises a tuning opportunity.

\section{COMPUTATIONAL DETAILS}

We performed a density functional theory calculations [27] using the projector-augmented wave (PAW) within the generalized gradient approximation (GGA) [28], and we equally used the PAW database from the pslibrary project [29]. The DFT is as implemented in the Quantum Espresso package [30]. The Perdew-Burke-Ernzehof (PBE) functional within the generalized gradient approximation (GGA) [28] is used for the exchange and correlation interaction. We did a structural optimization test for the conventional Wyckoff positions and three other Wyckoff positions by varying the lattice parameters between -0.5 and 0.5 in steps of 0.1 . The behavior of the alloys at equilibrium temperature in terms of the volume and pressure was calculated by fitting the result obtained from the total energy calculation to the Birch-Murnaghan equation of state [31-33]. The equilibrium kinetic energy cut-off and k-point mesh were subjected to the convergence test. Hence, all calculations were done using 70 and 700 for ecutwfc and ecutrho respectively. The system was treated in FCC crystallized phase. A Monkhorst-Pack [34] $10 \times 10 \times$ $10 \mathrm{k}$-point mesh was used for the SCF calculation. 
We used a denser grid of $20 \times 20 \times 20$ with a tetrahedraon-occupation for the density of states. For the band structure calculations, we selected dense high symmetry k-points for the FCC structure using XCrySDen. We used the stress-strain approach as implemented in the thermo_pw package [35] to compute the elastic constants and other mechanical properties of CoMnTiX(X = Sb and $S n)$. In this approach, two set of strains were applied $\mathrm{e}_{1}$ and $\mathrm{e}_{4}$. For each strain, a stress-strain calculation was performed ranging from -0.0075 to +0.0075 in steps of 0.005 . The results were interpolated by a quadratic polynomial and the values of the elastic constants $\mathrm{C}_{11}, \mathrm{C}_{12}$ and $\mathrm{C}_{44}$ were calculated from the coefficient of the linear term.

\section{RESULTS AND DISCUSSIONS}

\subsection{Structural Properties}

Most Heusler alloys crystallize in the cubic phase, specifically the face centered cubic (FCC) phase. Hence, both alloys were treated in that light. CoMnTiSn and CoMnTiSb belong to the space group $\mathrm{F} \overline{43} \mathrm{~m}$ (216). By substituting one of the $\mathrm{X}$-atoms in an $\mathrm{X}_{2} \mathrm{YZ}$ ternary Heusler alloy with a transition metal, we obtained the LiMgPdSb prototype structure $[7,8]$ that was found to be a well-ordered Y-type structure. The valency of the elements considered are (Co- $\left.3 d^{7} 4 s^{2}, M n-3 d^{5} 4 s^{2}, T i-3 d^{2} 4 s^{2}, S n-5 s^{2} 5 p^{2}, S b-5 s^{2} 5 p^{3}\right)$. Considering the valency, the most stable state of both materials was first ascertained by performing an optimization test for the three stoichiometric arrangements for a quaternary system.

The structural analysis of both crystal compounds under consideration was performed by considering the three possible structural configurations, which we define as type-Y1, type-Y2 and type-Y3. The atomic positions for all types are presented in Table 1, along with the total energy, lattice constant and the total magnetization. The Results show that both CoMnTiSn(Sb) are structurally stable in the type-Y1 configuration as ferromagnetic and non-magnetic systems respectively. The primitive cell structures as viewed by XCrySDen [36] showing the bonding for both alloys are presented in Fig. 1 (a \& b). The optimized equilibrium values were fitted to the Murnaghan equation of state as total energy versus the lattice constant, and these results are presented in Figs. 2. and 3. respectively. It is evident that ferromagnetism is favored in CoMnTiSn.

Several tests were performed to determine the stability of the structure in terms of its bonding and the Wyckoff positions of the atoms for the non-magnetic state, the ferromagnetic state and the antiferromagnetic states of both alloys. Interestingly, CoMnTiSb crystallizes as a non-magnetic system whereas CoMnTiSn crystallizes as a ferromagnetic system. There is no evidence of half metallicity in either alloys.

Table I.: Wyckoff positions for the three configurations considered, the lattice constants (a), the total magnetic moments $\left(M_{t}\right)$, the total energies and the magnetic states for the three $Y$ types of CoMnTiSn(Sb) considered.

\begin{tabular}{|l|l|l|l|l|l|l|}
\hline Alloy & Y-type & $\begin{array}{l}\text { Wyckoff } \\
\text { positions }\end{array}$ & a (a.u.) & $\mathrm{M}_{\mathrm{t}}(\mu \mathrm{B})$ & $\mathrm{E}_{\text {tot }}(\mathrm{Ry})$ & $\begin{array}{l}\text { Magnetic } \\
\text { state }\end{array}$ \\
\hline \multirow{3}{*}{ CoMnTiSn } & type-Y1 & $3 / 4,1 / 4,1 / 2,0.0$ & 11.584 & 2.06 & -1074.40189 & $\mathrm{FM}$ \\
\cline { 2 - 7 } & type-Y2 & $0.0,3 / 4,1 / 2,1 / 4$ & 11.773 & 4.00 & -1074.34827 & $\mathrm{FM}$ \\
\cline { 2 - 7 } & type-Y3 & $0.0,3 / 4,1 / 4,1 / 2$ & 11.622 & 4.37 & -1074.34459 & $\mathrm{FM}$ \\
\hline \multirow{3}{*}{ CoMnTiSb } & type-Y1 & $3 / 4,1 / 4,1 / 2,0.0$ & $11.433(11.471)$ & $0.0\left(1.0^{43}\right)$ & -980.00894 & $\mathrm{NM}\left(\mathrm{HMF}^{43}\right)$ \\
\cline { 2 - 7 } & type-Y2 & $0.0,3 / 4,1 / 2,1 / 4$ & 11.622 & 0.0 & -979.89644 & $\mathrm{NM}$ \\
\cline { 2 - 7 } & type-Y3 & $0.0,3 / 4,1 / 4,1 / 2$ & 11.622 & 0.0 & -979.91088 & $\mathrm{NM}$ \\
\hline
\end{tabular}




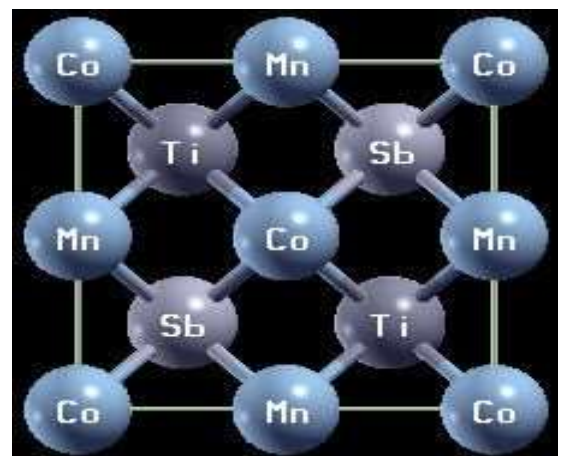

(a)

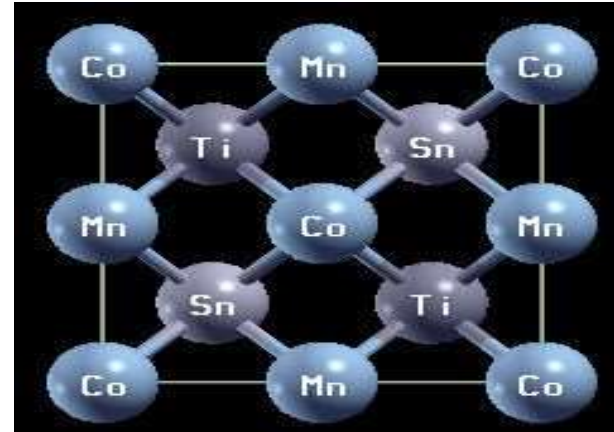

(b)

Fig. 1. Unit cell and bonding for (a) CoMnTiSb (b) CoMnTiSn viewed with Xcrysden

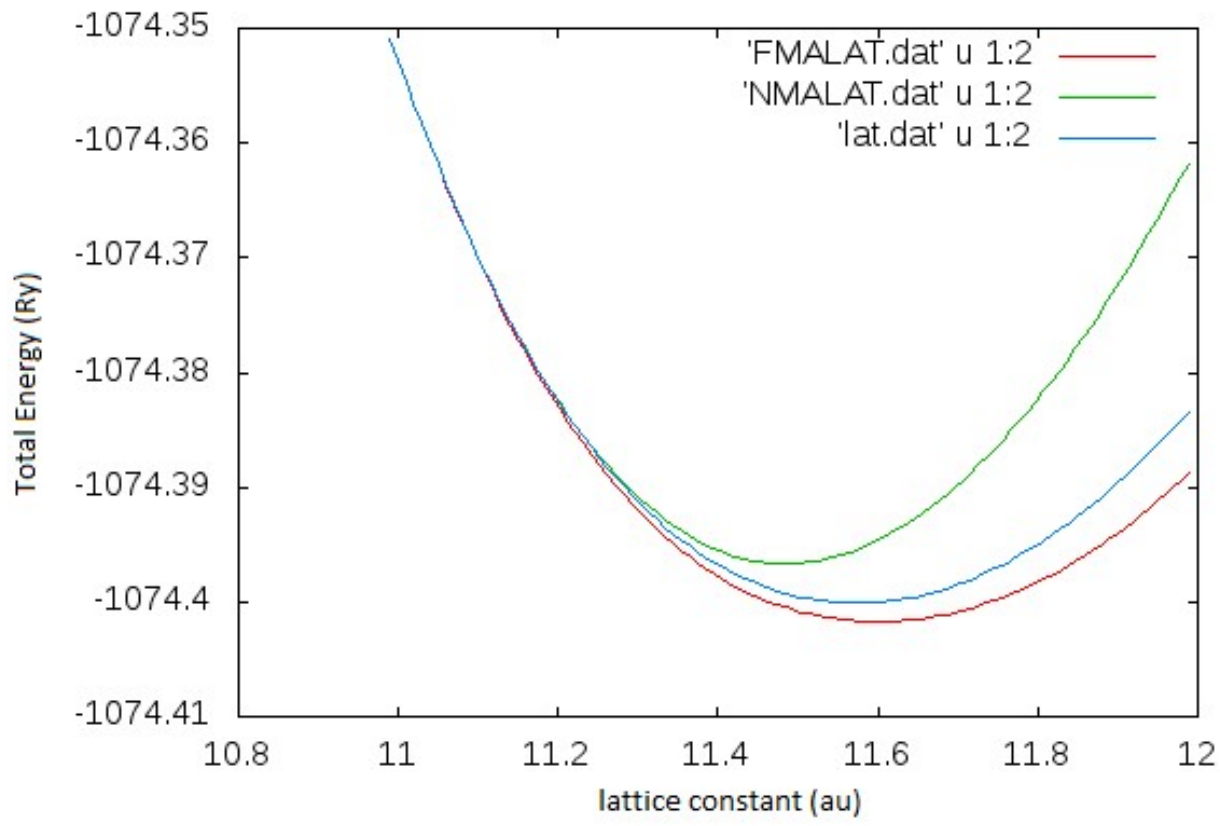

Fig. 2. Total energy (Ry) vs. lattice constants (au) for CoMnTiSn. The red, blue and green lines represent the ferromagnetic, antiferromagnetic and non-magnetice states, respectively. 


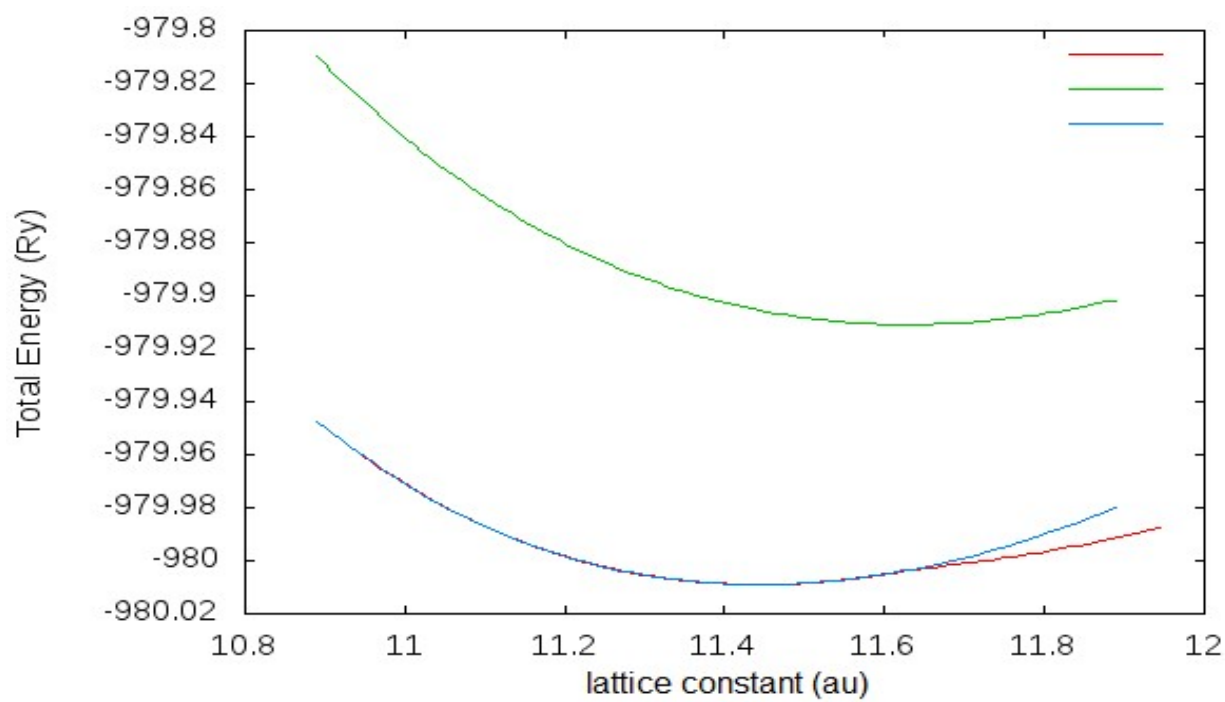

Fig. 3. Total energy (Ry) vs. lattice constants (au) for CoMnTiSb, the red, blue and green lines represent the ferromagnetic, antiferromagnetic and non-magnetic states for values between -0.5 to 0.5 of the lattice parameters

\subsection{Magnetic Properties}

The two compounds considered in this study behave uniquely, in that they violate the Slater-Pauling rule for half-metallic Heusler alloys. According to this rule, 24 valence electron compounds are expected to be semiconductors for example $\mathrm{Fe}_{2} \mathrm{Val}$ [37] hence, the possibility of half-metallic behavior is nil. The results show that CoMnTiSb with a valency of 25 is non-magnetic, in contrast to the expected value of $1.00 \mu \mathrm{B}$ in the most stable type-Y1 structure. This is probably due to the replacing of $\mathrm{Co}$ in $\mathrm{Co}_{2} \mathrm{MnSb}$ with $\mathrm{Ti}$ in the $\mathrm{X}^{\prime}$ position which leads to a de-positioning of the Van Hove singularity [38] causing a diversion in the density of states near the Fermi energy.

On the other hand, CoMnTiSn displays a total magnetization of 2.06, deviating from the value of zero predicted by the Slater-Pauling rule [2] and eliminating the possibility of half metallicity in this alloy as well. It is also clearly ferromagnetically ordered. Hence, we see a direct zero band gap with no overlap below the Fermi level in the majority band and a direct band gap of $0.4 \mathrm{eV}$ at the $\Gamma$ point above the Fermi level in the minority band making it a possible p-type spin gapless semiconductor. Experimentally, we believe this opens the possibility of tuning the band gap via doping to meet the desired need.

Considering that $\mathrm{CoMnTiSn}(\mathrm{Sb})$ are derived from the full Heusler alloys $\mathrm{Co}_{2} \mathrm{TiSn}(\mathrm{Sb})$ and $\mathrm{Co}_{2} \mathrm{MnSn}(\mathrm{Sb})$, the results are compared with the behavior of half-Heusler (111) CoTiSb, CoTiSn [39], CoMnSb and CoMnSn [9] as well as full Heusler (211) $\mathrm{Co}_{2} \mathrm{TiSb}$ [40], $\mathrm{Co}_{2} \mathrm{MnSb}$ [40] $\mathrm{Co}_{2} \mathrm{TiSn}$ [41] and $\mathrm{Co}_{2} \mathrm{MnSn}$ [41]. The results (in terms of the magnetization and magnetic states) are presented in Table 2. It is observed that materials with $\mathrm{Ti}$ in the $\mathrm{Y}$ position deviate from the Slater Pauling rule unlike the $\mathrm{Mn}$ in $\mathrm{Y}$ position materials. Considering the proper behavior of half and full Heusler CoMnSn(Sb) with respect to the Slater Pauling rule, the bonding of Co-Ti-Mn as shown in Figs. $1 \mathrm{a}$ and $1 \mathrm{~b}$ in the quaternary alloy seem not to energetically favor magnetization with the $\mathrm{Sb}$ main group element, whereas it is energetically favored with $\mathrm{Sn}$. The magnetic behavior can also be largely attributed to the s-p atoms hybridized with Co-Ti-Mn in the compounds. This goes a long way to show that the electronic configuration of the main group element has a great influence on the magnetic properties of the Heusler alloy even though it is largely non magnetic as an element. Both s-p elements used in this work are in group 5 and forms a tetrahedral bond with Co and $\mathrm{Mn}$, but they do not hybridize so this shifts the gap from the Fermi level. Furthermore, the most stable state finds the $\mathrm{Sb}$ and $\mathrm{Sn}$ atom in the tetrahedral positions. 
The short-range tetrahedral bonding of $\mathrm{Co}-\mathrm{Sn}(\mathrm{Sb})$ and $\mathrm{Mn}-\mathrm{Sn}(\mathrm{Sb})$ with a Ti atom enhances (destroys) magnetism and distorts the supposed well ordered magnetization of the alloys that is observed in $\mathrm{Co}_{2} \mathrm{MnSn}(\mathrm{Sb})$ and $\mathrm{CoMnSn}(\mathrm{Sb})$.

Table 2. Results from present calculations and literature. The Present work and experimental results from literature are represented by $\left({ }^{a}\right)$ and, ( $\left.{ }^{\exp }\right)$ respectively. The lattice constants (a), the total magnetic moment (Mt) per formula unit, the magnetic state, the energy band gap (gap) and the valence electrons are compared.

\begin{tabular}{|c|c|c|c|c|c|}
\hline & $\mathrm{a}(\mathrm{au})$ & Mt ( $\mu \mathrm{B} /$ f.u. $)$ & Mag. State & gap (eV) & $\mathrm{nv}$ \\
\hline CoMnTiSb & 11.433 & $0.01^{\mathrm{a}},\left(1.00^{43}\right)$ & $\mathrm{NSC}^{\mathrm{a}}\left(\mathrm{HMF}^{43}\right)$ & $0.4^{\mathrm{a}},\left(0.1^{43}\right)$ & 25 \\
\hline CoMnTiSn & 11.584 & $2.06^{\mathrm{a}}$ & $\mathrm{FM}^{\mathrm{a}}$ & - & 24 \\
\hline $\mathrm{CoMnSb}^{9}$ & & 3.00 & HMF & 0.888 & 21 \\
\hline $\mathrm{Co}_{2} \mathrm{TiSb}^{40}$ & & 1.24 & $\mathrm{M}$ & - & 27 \\
\hline CoMnSn ${ }^{9}$ & & 3.00 & HMF & 0.73 & 20 \\
\hline CoTiSn ${ }^{39}$ & & 0.97 & NSC & 0.865 & 17 \\
\hline $\mathrm{Co}_{2} \mathrm{TiSn}^{41}$ & 11.476 & 2.0002 & HMF & 0.243 & 26 \\
\hline $\mathrm{CoTiSb}^{39}$ & & 1.05(vasp) & NSC & & 18 \\
\hline $\mathrm{Co}_{2} \mathrm{MnSn}^{41}$ & $\begin{array}{l}11.338 \\
11.344 \exp 42\end{array}$ & $\begin{array}{l}5.043 \\
4.94 \exp \end{array}$ & HMF & & 29 \\
\hline $\mathrm{Co}_{2} \mathrm{MnSb}^{40}$ & $11.231 \exp 42$ & $\begin{array}{l}6.00 \\
5.09^{\exp }\end{array}$ & HMF & & 30 \\
\hline
\end{tabular}

\subsection{Electronic Properties}

We present results for the spin resolved band structures and the density of states in Figs. 4, 5, 6 and 7. A direct band gap of about $0.3 \mathrm{eV}$ is located above the Fermi level (conduction band) for CoMnTiSn in the minority spin sub band (Fig. 4.), whereas a zero band gap is observed at $\Gamma$ in the majority spin sub band just below the Fermi level (valence band) (Fig. 5). This compound with slight tuning behaves as a quasi ptype spin gapless semiconductor (SGS) as seen (Fig. 6) in the spin resolved total density of states (DOS).

The non-magnetic band structure of CoMnTiSb is calculated and the band structural result presented in Fig. 7, alongside the total density of states. The band structure displays a direct p-type band gap of 0.4 $\mathrm{eV}$ at the gamma point $(\Gamma)$ with respect to the Fermi level. This is contrary to the findings of Khodami and Ahmadian [43] who predicted it to be a half metallic ferromagnet with a band gap of $0.1 \mathrm{eV}$. The authors in Ref. 43 performed the density functional theory calculation using the self-consistent full-potential linearized augmented plane wave (FPLAPW) method. Their lattice constant is within the range of what we obtained using the quantum ESPRESSO code. They worked within a range of 5.46-6.22 $\AA$ for the lattice constant. The compression/expansion of the crystal alloy might have had a significant effect on the magnetic properties of the alloy. Our calculation was based on the most stable ground state properties of the alloys 


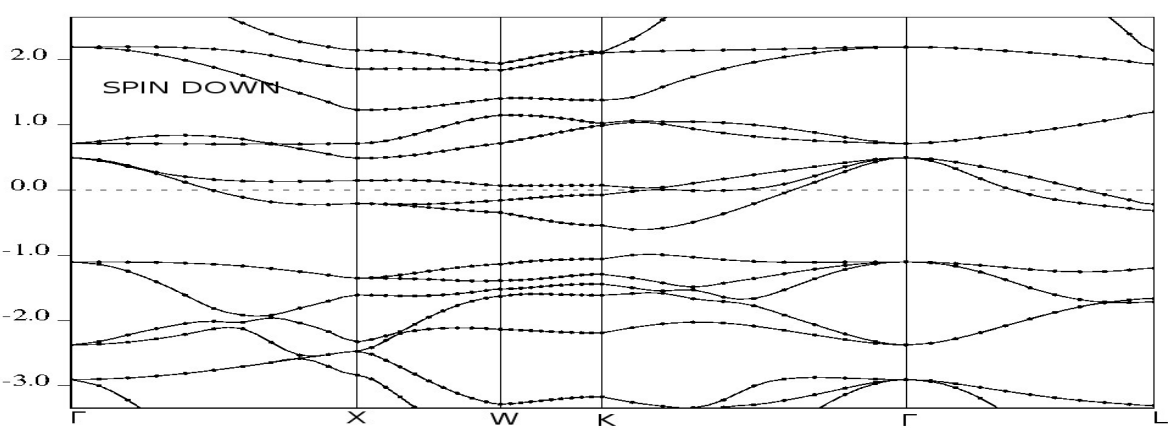

Fig. 4. Spin resolved band structure of the minority band of CoMnTiSn

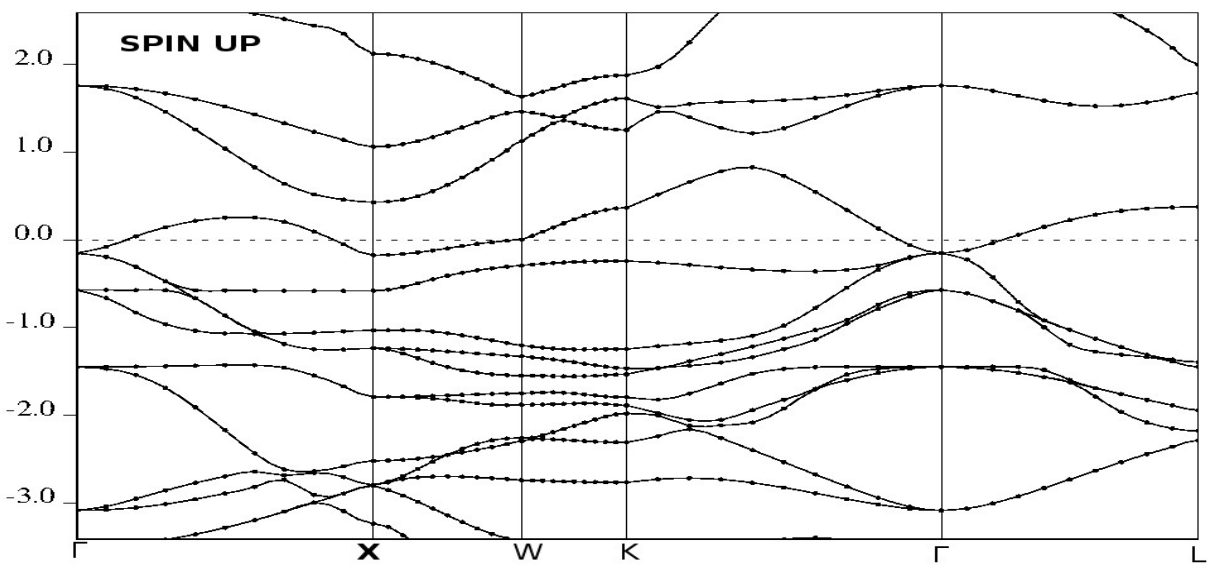

Fig. 5. Spin resolved band structure of the majority band of CoMnTiSn,

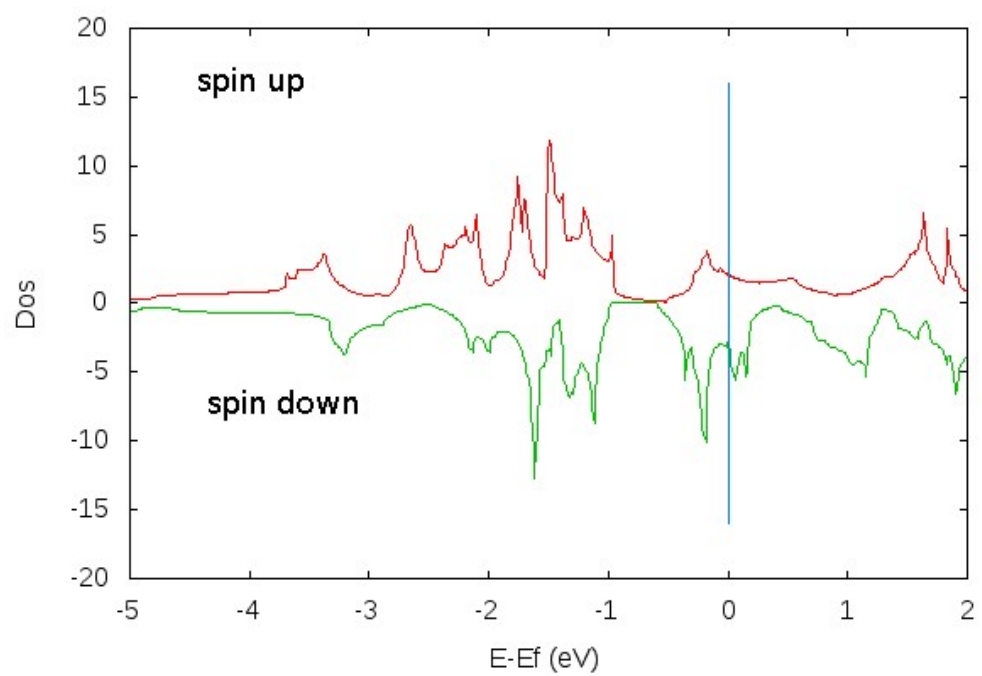

Fig. 6. Spin resolved total density of states of CoMnTiSn. The green and red lines represent the majority and minority bands, respectively. 


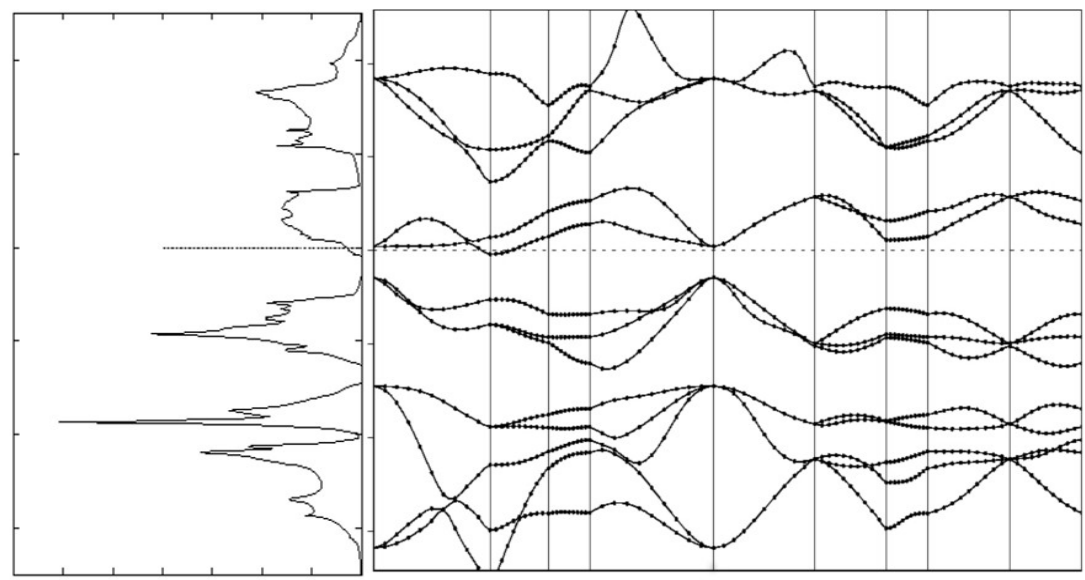

Fig. 7. Band structure and total density of states for non-magnetic CoMnTiSb. The dotted line shows the Fermi level for the band structure and the density of states

\subsection{Mechanical Properties}

The Heusler compounds have been studied structurally and electronically. The peculiarity of the behavior prompted us to study their mechanical stability. To do this, the elastic constants were calculated theoretically using the thermo-pw package [35] in interface with Quantum Espresso by the stress-strain approach. The values of the elastic constants $\mathrm{C}_{11}, \mathrm{C}_{12}$, and $\mathrm{C}_{44}$ obtained from the coefficient of the linear term are presented in Table 3 . The elastic stability of a cubic structure is determined by the following rules [44-46];

$$
\left.\begin{array}{c}
C+2 C \succ 0 \\
\varepsilon_{44}^{11} \succeq C_{12}^{12} \succ 0 \\
11
\end{array}\right\}
$$

These independent elastic constant parameters are of interest in the cubic phases [25, 47]. The values of the elastic constants of CoMnTiSb and CoMnTiSn are tabulated in Table 3 with calculated values of the Young's modulus $(Y)$, the Poisson ratio $(v)$ and the anisotropy factor $\left(A^{u}\right)$. By the Voigt-Reuss-Hill average approximation [48-50], the large values of the bulk modulus in kbar show that they are actually hard materials. To further buttress the brittleness (ductility) of the compounds, empirically, Pugh [51] says that the $\mathrm{B} / \mathrm{G}$ ratio for a material should be less (greater) than 1.75 for it to be brittle (ductile).

According to this rule, both compounds are ductile and hence, mechanically stable. For a layered crystal, the compounds are not expected to be isotropic, therefore the extent of the elasticity of the anisotropy was calculated. This was achieved using the Ranganathan \& Ostoja-Starzewski [52] universal index $A^{u}$ proposal for the calculation of the anisotropy. This is defined in Eqn. (2) as;

$$
A^{u}=5 \frac{G_{V}}{G_{R}}+\frac{B_{V}}{B_{R}}-6
$$

$B, G, V$, and $R$ are the bulk modulus, shear modulus, Voigt approximation [48] and Reuss approximation [49] respectively. As long as $A^{u}$ is not zero, the compound is not isotropic and the extent of deviation from zero is a measure of the elastic anisotropy of the compound. In this case, the values are 0.3059 for CoMnTiSb and 2.593 for CoMnTiSn. Showing that CoMnTiSn is a more elastic material. 
The stationary conditions of the Poisson ratio, given by Norris [53] as

$$
\left.\begin{array}{c}
S_{14}=0+S=0 \\
15=25 \\
(2 v-1) S_{16}+S_{26}=0
\end{array}\right\}
$$

are all satisfied. Therefore, both compounds are stable at the temperatures presented in Table 3 . This table also shows that CoMnTiSn(Sb) has a $56 \%(64 \%)$ possibility of resistance to deformation under compressive force.

Table 3 Elastic constants of CoMnTiSb and CoMnTiSn with calculated values of the Poisson ratio $(v)$, bulk modulus (B), shear modulus $(G)$, brittleness(ductility) ratio $(B / G)$, bulk Voigt approximation

\begin{tabular}{|c|c|c|c|c|c|c|c|c|c|c|}
\hline $\begin{array}{l}\text { Compoun } \\
\text { d }\end{array}$ & $V$ & $\begin{array}{c}\mathrm{B} \\
\text { (kbar) }\end{array}$ & $\begin{array}{c}G \\
\text { (kbar } \\
\text { ) }\end{array}$ & $\mathrm{B} / \mathrm{G}$ & $\begin{array}{c}\mathrm{B}_{V} \\
\text { (kbar) }\end{array}$ & $\begin{array}{c}\mathrm{B}_{R} \\
\text { (kbar) }\end{array}$ & $\begin{array}{c}\mathrm{G}_{V} \\
\text { (kbar } \\
\text { ) }\end{array}$ & $\begin{array}{c}\mathrm{G}_{R} \\
\text { (kbar } \\
\text { ) }\end{array}$ & $\begin{array}{c}Y \\
\text { (kbar) }\end{array}$ & $\begin{array}{l}\text { Dyb. } \\
T(K)\end{array}$ \\
\hline CoMnTiSb & 0.28 & $\begin{array}{l}100.9 \\
1\end{array}$ & 50.59 & 1.99 & $\begin{array}{l}100.9 \\
1\end{array}$ & $\begin{array}{l}100.9 \\
1\end{array}$ & 61.01 & 40.17 & $\begin{array}{l}129.3 \\
7\end{array}$ & $\begin{array}{l}340.5 \\
2\end{array}$ \\
\hline & & $\begin{array}{l}188.6 \\
9\end{array}$ & & & $\begin{array}{l}188.6 \\
9\end{array}$ & $\begin{array}{l}188.6 \\
9\end{array}$ & & 75.48 & $\begin{array}{l}205.1 \\
5\end{array}$ & \\
\hline
\end{tabular}
$\left(B_{V}\right)$ and bulk Reuss approximation $\left(B_{R}\right)$, shear Voigt approximation $\left(G_{V}\right)$, shear Reuss approximation $\left(G_{R}\right)$, Young's modulus $(Y)$, and the Debye temperature (Dyb. $T$ )

\section{CONCLUSION}

In this work, we have performed first principles calculations of the structural, magnetic, electronic and mechanical properties of the quaternary CoMnTiSn and CoMnTiSb Heusler compounds. We performed all calculations using the GGA exchange correlation functional. To the best of our knowledge, this is the first report for the CoMnTiSn compound, whereras our result for the magnetic moment of CoMnTiSb differs from what was reported by Khodami and Ahmadian using the self-consistent full potential linearized augmented plane wave (FPLAPW) method. However, the lattice constants obtained in these two studies are in agreement. CoMnTiSb is a ferromagnetic semiconductor with a band gap of $0.4 \mathrm{eV}$, whereas CoMnTiSn is almost p-type spin gapless semiconductor. There is no previous experimental work except for that on Co2TiSb, which also violates the Slater-Pauling rule. The calculated elastic constant shows that both compounds are hard but ductile materials. There are also indications that they are mechanically stable with a $56 \% / 65 \%$ resistance to deformation for $\mathrm{CoMnTiSb}(\mathrm{Sn})$. These materials can be experimentally stimulated to explore their application to semi conductor spintronic devices. 


\section{REFERENCES}

[1] C. Chappert, A. Fert, F.N.V. Dau, Nat. Mater. 6 (2007) 813. DOI: 10.1038/nmat2024

[2] C. Felser, G.H. Fecher, B. Balke, Angew. Chem. Int. Ed. 46 (2007) 668. DOI: 10.1002/anie.200601815

[3] K. Inomata, N. Ikeda, N. Tezuka, R. Goto, S. Sugimoto, M. Wojcik, E. Jedryka, Sci. Technol. Adv. Mater 9 (2008) 014101. DOI: 10.1088/1468-6996/9/1/014101

[4] R. A. De Groot, F. M. Müller, P. G. Van Engen, and K. H. J. Buschow, Phys. Rev. Lett. 50, 2024 (1983). DOI: https://10.1103/PhysRevLett.50.2024

[5] H. Akai, Phys. Rev. Lett. 81, 3002 (1998).

[6] S. P. Lewis, P. B. Allen, and T. Sasaki, Phys. Rev. B 55, 10253 (1997).

[7] U. Eberz, W. Seelentag, and H. U. Schuster, Z. Naturforsch. B 35 (1980) 1341.

[8] H. Pauly, A. Weiss and H. Witte, Z. Metallkunde 59 (1968) 47.

[9] K. Ozdogan, E. Sasioglu, and I. Galanakis, J. Appl. Phys. 113, 193903 (2013). DOI: http://dx.doi.org/10.1063/1.4840318

[10] X. Dai, G. Liu, G. H. Fecher, C. Felser, Y. Li, and H. Liu, J. Appl. Phys. 105, 07E901 (2009). https://doi.org/10.1063/1.3062812

[11] S. Ouardi, G. H. Fecher, C. Felser and Jürgen. Kübler, Phys. Rev. Lett. 110, 100401 (2013). DOI: https://doi.org/10.1103/PhysRevLett.110.100401

[12] G. Z. Xu, E. K. Liu, Y. Du, G. J. Li, G. D. Liu,W. H.Wang, and G. H. Wu, Europhys. Lett. 102(1), 17007 (2013).

[13] G. Y. Gao and K. L. Yao, Appl. Phys. Lett. 103, 232409 (2013). http://dx.doi.org/10.1063/1.4840318

[14] S. Skaftouros, K. Ozdogan, E. Sasioglu, and I. Galanakis, Appl. Phys. Lett. 102, 022402 (2013). DOI: https://doi.org/10.1103/PhysRevB.87.024420

[15] Lakhan Bainsla, A. I. Mallick, M. Manivel Raja, A. K. Nigam, B.S.D.Ch.S. Varaprasad, Y. K. Takahashi, Aftab Alam, K. G. Suresh, K. Hono, Phys. Rev. B 91, 104408 (2015). DOI: https://doi.org/10.1103/PhysRevB.91.104408

[16] G. Z. Xu, Y. Du, X. M. Zhang, H. G. Zhang, E. K. Liu, W. H. Wang, and G. H. Wu, Appl. Phys. Lett. 104, 242408 (2014). DOI: https://doi.org/10.1063/1.4884203

[17] M. E. Jamer, B. A. Assaf, T. Devakul, and D. Heiman, Appl. Phys. Lett. 103, 142403 (2013). https://doi.org/10.1063/1.4823601

[18] M. Wang, R. P. Campion, A. W. Rushforth, K. W. Edmonds, C. T. Foxon, and B. L. Gallagher, Appl. Phys. Lett. 93, 132103 (2008). DOI: https://doi.org/10.1063/1.2992200

[19] X. L. Wang, Phys. Rev. Lett. 100, 156404 (2008). DO1: https://doi.org/10.1103/PhysRevLett.100.156404

[20] B. Balke, S. Wurmel, G. H. Fecher, C. Felser, M. C. M. Alves, F. Bernardi and J. Morais, Appl. Phys. Lett. (2007), 90, 172501. DOI: https://doi.org/10.1063/1.2731314

[21] V. Alijani et al., Phy. Rev. B 84, 224416 (2011). DOI: https://doi.org/10.1103/PhysRevB.84.224416

[22] P. Klaer, B. Balke, V. Alijani, J. Winterlik, G.H. Fecher, C. Felser, H.J. Elmers, Phys.

Rev. B 84 (2011) 144413. DOI: https://doi.org/10.1103/PhysRevB.84.144413

[23] Dinh V A, Sato K and Katayama-Yoshida H 2008 arXiv: 0801.2222

[24] J.P. Perdew, A. Zunger, Phys. Rev. B 23 (1981) $5048 . \quad$ DOI: https://doi.org/10.1103/PhysRevB.23.5048

[25] V. Alijani, J. Winterlik, G.H. Fecher, S.S. Naghavi, C. Felser, Phys. Rev. B 83 (2011) 184428. DOI: https://doi.org/10.1103/PhysRevB.84.184428

[26] T. M. Bhat and D. C. Gupta, RSC Adv., 2016, DOI: 10.1039/C6RA18934B.

[27] W. Kohn, L.J. Sham, Phys. Rev. 140 (1965) A1133. DOI: https://doi.org/10.1103/PhysRev.140.A1133

[28] J.P. Perdew, A. Zunger, Phys. Rev. B 23 (1981) 5048

[29] http://qe-forge.org/gf/project/pslibrary.

[30] P. Giannozzi, et al., J. Phys.: Condens. Matter 21 (2009) 395502

[31] F. D. Murnaghan, Proc. Natl. Acad. Sci, 30, 244 (1944)

[32] F. Birch, Phys. Rev. 71, 809 (1947) 
[33] F. D. Murnaghan, Am. J. Math. 49, 235 (1937)

[34] H.J. Monkhorst, J.D. Pack, Phys. Rev. B 13 (1976) 5188.

[35] www.qe-forge.org/gf/project/thermopw/.

[36] A. Kokalj, Comp. Mater. Sci., 28,155-168 (2003). DOI: https://doi.org/10.1016/S09270256(03)00104-6

[37] P. J. Webster and K. R. A. Ziebeck, Physics Letters A,98(1,2), 51 - 53 (1983). DOI: https://doi.org/10.1016/0375-9601(83)90543-1

[38] L. Van Hove, Phys. Rev. 89, 1189-1193 (1953).

[39] Jianhua Ma, Vinay I Hegde, Kamaram Munira, yunkun xie, Sahar keshavarz, David T, Mildebrath, C. Wolverton, Avik W. Ghosh and W. H Butler (2016) arxiv:1610.02444v2

[40] D.P Rai and R.K. Thapa (2013) Chinese journal of physics vol 51(4) Dol:10.6122/51(4)812

[41] Barth, Joachim and Fecher, Gerhard H. and Balke, Benjamin and Graf, Tanja and Shkabko, Andrey and Weidenkaff, Anke and Klaer, Peter and Kallmayer, Michael and Elmers, HansJoachim and Yoshikawa, Hideki and Ueda, Shigenori and Kobayashi, Keisuke and Felser, Claudia. Philosophical Transactions of the Royal Society of London A: Math., Physical and Engr. Sci., 369, 3588--3601 (2011). DOI: https://doi.org/10.1098/rsta.2011.0183

[42] Moti R. Paudel, Christopher S. Wolfe, Heather Patton, Igor Dubenko, Naushad Ali, Joseph A. Christodoulides, and Shane stadler.(2009) Journal of Appl. Phys. 105, 013716. DOI: https://doi.org/10.1063/1.3054291

[43] M. Khodami, and F. Ahmadian. J. Supercond. Nov. Magn. 28, 3027(2015) 28: 3027. DOI: https://doi.org/10.1007/s10948-015-3126-2

[44] Félix Mouhat and François-Xavier Coudert, Phys. Rev. B 90, 224104 (2014). DOI: https://doi.org/10.1103/PhysRevB.90.224104

[45] M. Born, K. Huang, Dynamical Theory of Crystal Lattices, Clarendon Press, Oxford, 1956.

[46] J.F. Nye, Physical Properties of Crystals, Oxford Science Publications, Oxford, 1985.

[47] O. E. Osafile, P. O. Adebambo and G. A. Adebayo, J. Alloys and Compds, 722, 207-211 (2017). http://dx.doi.org/10.1016/j.jallcom.2017.05.338

[48] W. Voigt, Lehrbuch der Kristallphysik, (Teubner and Leipzig), p. 739 (1928)

[49] A. Reuss, Z. Angew. Math. Mech. 9, 49 (1929)

[50] R. Hill, Proc. Phys. Soc. (London) 65, 349 (1952)

[51] Pugh, S. F., Philos. Mag. 45, 823-843 (1954)

[52] Shivakumar I. Ranganathan and Martin Ostoja-Starzewski, Phys. Rev. Lett. 101,055504 (2008)

[53] A. N. Norris, Q. J.Mech. Appl. Math., 59(1), 29-53 (2006) 